\title{
Necessary Conditions for a Fixed Point of Maps in Non-Metric Spaces
}

\author{
I. Raykov \\ Department of Mathematics and Physics, Colorado State University, Pueblo, USA \\ Email: ivan.raykov@colostate-pueblo.edu
}

Received July 17, 2012; revised September 3, 2012; accepted September 11, 2012

\begin{abstract}
The main purpose of the present work is to introduce necessary conditions for a map on a non-metric space, defined by using a map on a metric space, to have a fixed point.
\end{abstract}

Keywords: Topological Space; Complete Metric Compact Space; Cauchy Sequence; Lipschitz Continuous Map; Contraction Map

\section{Introduction}

Let $X$ denote a complete (or compact) metric space and also $f: X \rightarrow Y$ a continuous map of $X$ onto $Y$, where $Y$ is a bounded closed topological normal space with a countable base.

What must be the conditions, in the means of the meric space $X$, such that the continuous map $g: Y \rightarrow Y$ from $Y$ onto $Y$ will have a fixed point?

We suppose that (see [1-3]):

the continuous map $f: X \rightarrow Y$ (not one to one) and

the continuous map $g: Y \rightarrow Y$ are given and

the continuous inverse map of $f, f^{-1}: Y \rightarrow X$ exists.

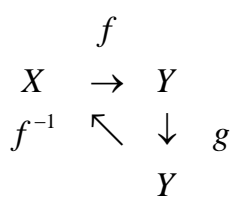

We remind that Banach contraction principle for multivalued maps is valid and also the next Theorem, proved by H. Covitz and S. B. Nadler Jr. (see [4]).

Theorem 1. Let $(X, d)$ be a complete metric space and $F: X \rightarrow B(X)$ a conraction map ( $B(X)$ denotes the family of all nonempty closed bounded (compact) subsets of $X$ ). Then there exists $x \in X$ such that $x \in F(x)$.

\section{Main Result}

We consider now the next theorem:

Theorem 2. Let $X$ denote a complete (or compact) metric space $X$ and also:

$f: X \rightarrow Y$ a continuous map of $X$ onto $Y$, where $Y$ is a bounded closed topological normal space with a countable base.

We suppose also that the maps:

$g: Y \rightarrow Y$ is continuous and onto. and

$f^{-1}: Y \rightarrow X$ exists and it is continuous.

If $x_{0} \in X$ is a point from $X$ and if we suppose also that $y_{0} \in f\left(x_{0}\right)$.

Then if the rest terms of the sequence $\left\{y_{i}\right\}$ are received from $y_{i} \in g\left(y_{i-1}\right), i=1,2,3, \cdots$ and the rest of the terms of the sequence $\left\{x_{i}\right\}$ are determined by $x_{i} \in f^{-1}\left(y_{i}\right), i=1,2,3, \cdots$ and if also $\left\{x_{i}\right\}$ is a Cauchy sequence and therefore convergent to a fixed point $x^{*}$ in $X$, then the sequence $\left\{y_{i}\right\}$ will be also convergent to a fixed point $y^{*}$ in $Y$.

Proof. Let $x_{0} \in X$ is a point from $X$ and let us suppose also that $y_{0} \in f\left(x_{0}\right) \subset Y$ and let the rest terms of the sequence $\left\{y_{i}\right\}$ are received from $y_{i} \in g\left(y_{i-1}\right)$, $i=1,2,3, \cdots$.

Let also the rest of the terms of the sequence $\left\{x_{i}\right\}$ are determined by $x_{i} \in f^{-1}\left(y_{i}\right), \quad i=1,2,3, \cdots$.

If $\left\{x_{i}\right\}$ is a Cauchy sequence then for any $\varepsilon>0$ there exists an integer $N_{\varepsilon}$, such that for all integers $i$ and $k, i>N_{\varepsilon}$ and $k>N_{\varepsilon}$ will be satisfied the inequality

$$
\left\|x_{i}-x_{k}\right\|<\varepsilon
$$

and therefore the Cauchy sequence $\left\{x_{i}\right\}$ will be convergent with a fixed point $x^{*}$ in $X$, and because $X$ is complete (or compact), i.e.

$$
\lim _{x \rightarrow \infty} x_{i}=x^{*}
$$

Since $x_{i}=f^{-1}\left(y_{i}\right)$ and $x_{i}=f^{-1}\left(y_{k}\right)$ and $f^{-1}(y)$ is a continuous map and $g(y)$ is continuous map onto 
the closed and bounded space $Y$, and also $y_{i} \in f\left(x_{i}\right)$ and $y_{k} \in f\left(x_{k}\right)$, therefore the sequence $\left\{y_{i}\right\}$ will be also convergent with a fixed point $y^{*}$ in $Y$, such that $x^{*}=f^{-1}\left(y^{*}\right)$ and $y^{*} \in f\left(x^{*}\right)$, i.e.

$$
\lim _{x \rightarrow \infty} y_{i}=y^{*} \text {. }
$$

Q.E.D.

\section{Acknowledgements}

We express our gratitude to Professor Alexander Arhangelskii from OU-Athens for creating the problem and to Professor Jonathan Poritz and Professor Frank Zizza from CSU-Pueblo for the precious help for solving this problem, and to Professor Darren Funk-Neubauer and Professor Bruce Lundberg for correcting some gram- matical and spelling errors.

\section{REFERENCES}

[1] A. V. Arhangel'skii and V. V. Fedorchuk, "The Basic Concepts and Constructions of General Topology,” In: A. V. Arkhangel'skii and L. S. Pontrjagin, Eds., General Topology I, Encyclopedia of the Mathematical Sciences, Springer, Berlin, 1990.

[2] J. Dugundji, “Topology,” Allyn and Bacon, Inc., Boston, 1966.

[3] S. W. Davis, “Topology,” McGraw-Hill, Boston, 2005.

[4] L. Gorniewicz, "Topological Fixed Point Theory of Multivalued Mappings,” Springer Verlag, Berlin, 2006. 